\title{
Longterm optical monitoring of bright BL Lacertae objects with ATOM: Spectral variability and multiwavelength correlations
}

\author{
Alicja Wierzcholska ${ }^{1,2,3}$, Michał Ostrowski ${ }^{1}$, Łukasz Stawarz $^{4,1},{\text { Stefan } \text { Wagner }^{3} \text {, and Marcus Hauser }}^{3}$ \\ 1 Astronomical Observatory, Jagiellonian University, ul. Orla 171, 30-244 Kraków, Poland \\ e-mail: alicja.wierzcholska@ifj.edu.pl \\ 2 Institute of Nuclear Physics, Polish Academy of Science, ul. Radzikowskiego 152, 31-342 Kraków, Poland \\ 3 Landessternwarte, Universität Heidelberg, Königstuhl, 69117 Heidelberg, Germany \\ ${ }^{4}$ Institute of Space and Astronautical Science JAXA, 3-1-1 Yoshinodai, Chuo-ku, Sagamihara, 252-5210 Kanagawa, Japan
}

Received 9 April 2014 / Accepted 7 September 2014

\begin{abstract}
Context. Blazars are the established sources of an intense and variable non-thermal radiation extending from radio wavelengths up to high and very high-energy $\gamma$-rays. Understanding the spectral evolution of blazars in selected frequency ranges, as well as multifrequency correlations in various types of blazar sources, is of a primary importance for constraining blazar physics.

Aims. We present the results of a long-term optical monitoring of a sample of 30 blazars of the BL Lac type, most of which are the confirmed $\mathrm{TeV}$ emitters. We study the optical color-magnitude correlation patterns emerging in the analyzed sample and compare the optical properties of the targets with the high-energy $\gamma$-ray and high-frequency radio data.

Methods. The optical observations were carried out in $R$ and $B$ filters using the Automatic Telescope for Optical Monitoring (ATOM) located at the site of the H.E.S.S. Array. Each object in the sample was observed during at least 20 nights in the period 2007 to 2012. Results. We find significant overall color-magnitude correlations (meaning bluer-when-brighter spectral evolution) in $40 \%$ of the sample. The sources that do not display any clear chromatism in the full datasets often do exhibit bluer-when-brighter behavior but only in isolated shorter time intervals. We also discovered spectral state transitions at optical wavelengths in several of the analyzed sources. Finally, we find that the radio, optical, and $\gamma$-ray luminosities of the sources in the sample obey almost linear correlations, which seem induced, at least partly, by the redshift dependence and may also be affected by the non-simultaneity of the analyzed multifrequency dataset.

Conclusions. We argue that the observed bluer-when-brighter behavior is intrinsic to the jet-emission regions, at least for some of these blazars, rather than resulting from the contamination of the measured flux by the starlight of host galaxies. We also conclude that the significance of color-magnitude scalings does not correlate with the optical color, but instead seems to depend on the source luminosity, in that these are the lowest luminosity BL Lac objects to display the strongest correlations.
\end{abstract}

Key words. radiation mechanisms: non-thermal - galaxies: active - BL Lacertae objects: general - galaxies: jets

\section{Introduction}

Blazars are radio-loud active galactic nuclei (AGN) with relativistic jets pointing at small angles to the line of sight (e.g., Begelman et al.1984). The Doppler-boosted non-thermal emission of these sources is observed in a wide range of frequencies of the electromagnetic spectrum from radio to X-rays and, in the case of the brightest objects, up to high- and very highenergy $\gamma$-rays (e.g., Ackermann et al. 2011 1 The rapid variability of blazars is routinely detected at different wavelengths on timescales down to hours or even minutes, albeit with often drastically different amplitudes (e.g., Wagner \& Witzel 1995; Aharonian et al. 2007; Sasada et al. 2008, Saito et al. 2013). Based on the optical classification, blazars can be divided into two subclasses: flat spectrum radio quasars (FSRQs) and BL Lacertaetype objects (BL Lac objects). The optical/UV spectra of FSRQs are characterized by the presence of prominent broad and narrow emission lines; BL Lac objects are instead dominated by continuum emission in the optical band (Urry \& Padovani 1995). BL Lac objects can be subdivided further into high-, intermediate-, and low-energy peaked sources (HBLs, IBLs, and

\footnotetext{
${ }^{1}$ See also http://tevcat.uchicago.edu
}

LBLs, respectively), depending on the position of their synchrotron peak frequencies (see, e.g., Padovani \& Giommi 1995, Fossati et al. 1998, Abdo et al. 2010).

The broad-band spectral energy distribution (SED, meaning the $v-v F_{v}$ spectral representation) of blazars has a characteristic double-hump structure. The first hump extends from radio up to the optical or X-ray ranges and is due to the synchrotron emission of in situ accelerated jet electrons, and the second hump is located in the $\gamma$-ray part of the spectrum and is widely believed to be due to the inverse-Compton emission of the same electron population (e.g., Konigl 1981; Marscher \& Gear 1985; Dermer \& Schlickeiser 1993, Sikora et al. 1994). The most recent multiwavelength campaigns targeting the brightest or dramatically flaring blazars have, to some extent, challenged the simplest (homogeneous one-zone) versions of this standard leptonic model, but truly simultaneous, broad-band, and long-term monitoring data for a representative sample of the blazar population, which are very important for constraining the blazar physics, are still relatively sparse.

Previous observational studies of blazars (BL Lac objects, in particular) at optical frequencies revealed complex flux-color correlation patterns. For example, the ten-year photometric 
monitoring of optically bright OJ 287 and BL Lacertae presented by Carini et al. (1992) did not indicate any universal and persistent relation between spectral and flux changes, although some hints of a general trend of the sources to appear bluer when brighter were noted. Interestingly, Carini et al. detected a microvariability in both blazars, at the level of $0.08 \mathrm{mag} / \mathrm{h}$ for OJ 287 and $0.01 \mathrm{mag} / \mathrm{h}$ for BL Lacertae. In a different dataset, a strong correlation between $V-R$ color and $R$ magnitude has been found in 11 nights of the optical outburst of BL Lacertae in 1997, with the correlation coefficient $\simeq 0.7$ (Clements \& Carini 2001). The analysis of the longterm monitoring of the same object by Villata et al. (2002, 2004) confirmed that bluer-when-brighter relation is hardly present in long-timescale variations but in short isolated outbursts. An analogous color evolution of OJ 287 during the flaring state in the years 2005-2006 was reported by Dai et al. (2011), who found the bluer-when-brighter chromatism for the source with the correlation coefficient $\simeq 0.67$.

Similar results regarding color-magnitude (CM) correlations - namely, clear bluer-when-brighter behavior during the rapid flares and basically achromatic longterm variability - were also reported for the another exceptionally bright and active (at optical frequencies) blazar S5 0716+714 (Dai et al. 2013, Ghisellini et al. 1997, Gu et al. 2006). We note that this source is known for its persistent variability down to the timescales of hours and minutes, both in total and in polarized optical fluxes (Sasada et al. 2008; Dai et al. 2013; Bhatta et al. 2013).

Besides the three aforementioned well-known BL Lac objects, the positive CM correlations were also detected in, e.g., 3C 66A (Ghosh et al. 2000), AO 0235+164 (Raiteri et al. 2001), and PKS 0735+178 (Gu et al. 2006). More recently, Ikejiri et al. (2011) presented a rich dataset gathered with the KANATA telescope from 2008 until 2010 for a sample of 42 blazars including both FSRQs and BL Lac objects. The authors established that the majority of the studied sources ( $88 \%$ of the sample) do exhibit a universal bluer-when-brighter trend in the optical band, with only a few exceptions, mostly FSRQs, which reveal the opposite redder-when-brighter behavior (see in this context also Bonning et al.2012, for the results of the SMARTS optical and infrared blazar monitoring program).

In addition to the CM correlation patterns, the optical polarization variability of bright blazars is now being widely studied (e.g., Sasada et al. 2008, Ikejiri et al. 2011; Gaur et al. 2014). The successful operation of the Large Area Telescope (LAT; Atwood et al.2009) onboard the Fermi satellite enabled, on the other hand, the first in-depth investigations of the optical $/ \gamma$-ray correlations for a large number of blazar sources detected on a daily basis at GeV energies. The extensive optical monitoring of the selected BL Lac objects revealed in particular significant flux-flux correlations during the flaring states (e.g., Raiteri et al. 2013 for the GASP-WEBT campaign on BL Lacertae; see also Chatterjee et al. 2012), as well as the overall optical and $\gamma$-ray luminosity correlations for the LAT-detected objects (Hovatta et al. 2014).

In this paper, we present the data gathered with the Automatic Telescope for Optical Monitoring (ATOM; Hauser et al. 2004) during the past six years (2007-2012) for a sample of 30 blazars of the BL Lac type. The targets were selected in the southern hemisphere using various criteria, such as promising $\mathrm{TeV}$ candidates or already established TeV emitters. We study the $B-R$ color versus $R$ magnitude correlations, along with the radio, optical, and GeV luminosity-luminosity or luminosityspectral scalings and dependencies. We emphasize that the analyzed multiwavelength dataset is not simultaneous, the discussed blazar sample is not complete, and the source light curves are not uniformly sampled with ATOM. Still, several general trends and correlation patterns are revealed, allowing for new insight into the variability properties of bright BL Lac objects.

\section{Data analysis and results}

\subsection{ATOM observations and target selection}

ATOM is the 75-cm optical telescope (see Hauser et al. 2004), which has been operating in Namibia since 2006 at the site of the High Energy Stereoscopic System (H.E.S.S.; see Aharonian et al. 2006). The telescope works in a fully robotic way in four filters in the Johnson-Cousins $U B V R I$ broad-band photometric system defined by Bessell (1990): $B(440 \mathrm{~nm}), V$ $(550 \mathrm{~nm}), R(640 \mathrm{~nm})$, and $I(790 \mathrm{~nm})$. The main scientific goal of ATOM observations is an automatic monitoring of $\gamma$ ray sources in the southern hemisphere, and in particular of the potential H.E.S.S. targets. The durations of single pointings analyzed in this paper are between $100 \mathrm{~s}$ and $1000 \mathrm{~s}$, and this enables us to limit the uncertainty of each single observation to below $0.1 \mathrm{mag}$. The photometric flux scale was calibrated using the reference stars. In addition to the standard automatic analysis of the collected data, some of the raw images were also checked manually, and a limited number of bad-quality pointings (e.g., due to bad weather conditions or a moon light contamination) were rejected from the final dataset. This process of discarding some of the observations does not affect the final results presented below. The observed magnitudes have been corrected against the Galactic extinction based on the model by Schlegel et al. (1998) with the most recent recalibration by Schlafly \& Finkbeiner (2011

The analyzed blazar sample includes 30 sources of the BL Lac type (mostly HBLs), all monitored frequently with the ATOM telescope, majority of which (21) are the established TeV emitters. In particular, we require each target in the sample to be observed for at least 20 - but not necessarily consecutive nights, simultaneously in $B$ and $R$ filters, in a time span from 2007 to 2012. When a given source was observed more than once during the same night, the average value of the pointings was used. A list of thus selected objects is given in Table 1 . and the particular dates of the ATOM observations are given in Table 2.

\subsection{Color-magnitude diagrams}

Figures $1-5$ present the $B-R$ colors vs $R$ magnitudes for the analyzed 30 blazars from our sample listed in Table 1 . For each object, we evaluate the corresponding CM Pearson's correlation coefficient $C_{(B-R, R)}$, taking all the available datapoints into account, in order to check and to quantify general trends in a spectral appearance at different flux levels. The obtained values of $C_{(B-R, R)}$, together with the emerging slopes of the regression fits, the average colors $\langle B-R\rangle$, and the corresponding spectral indices, are all given in Table 1 as well. The average spectral indices are derived here simply as

$\left\langle\alpha_{B R}\right\rangle=\frac{0.4\langle B-R\rangle}{\log \left(v_{B} / v_{R}\right)}$,

where $v_{B}$ and $v_{R}$ are effective frequencies of the respective bands (Bessell et al. 1998).

\footnotetext{
$\overline{2}$ See the corresponding $A_{B}$ and $A_{R}$ extinction coefficients collected in Table 1 .
} 


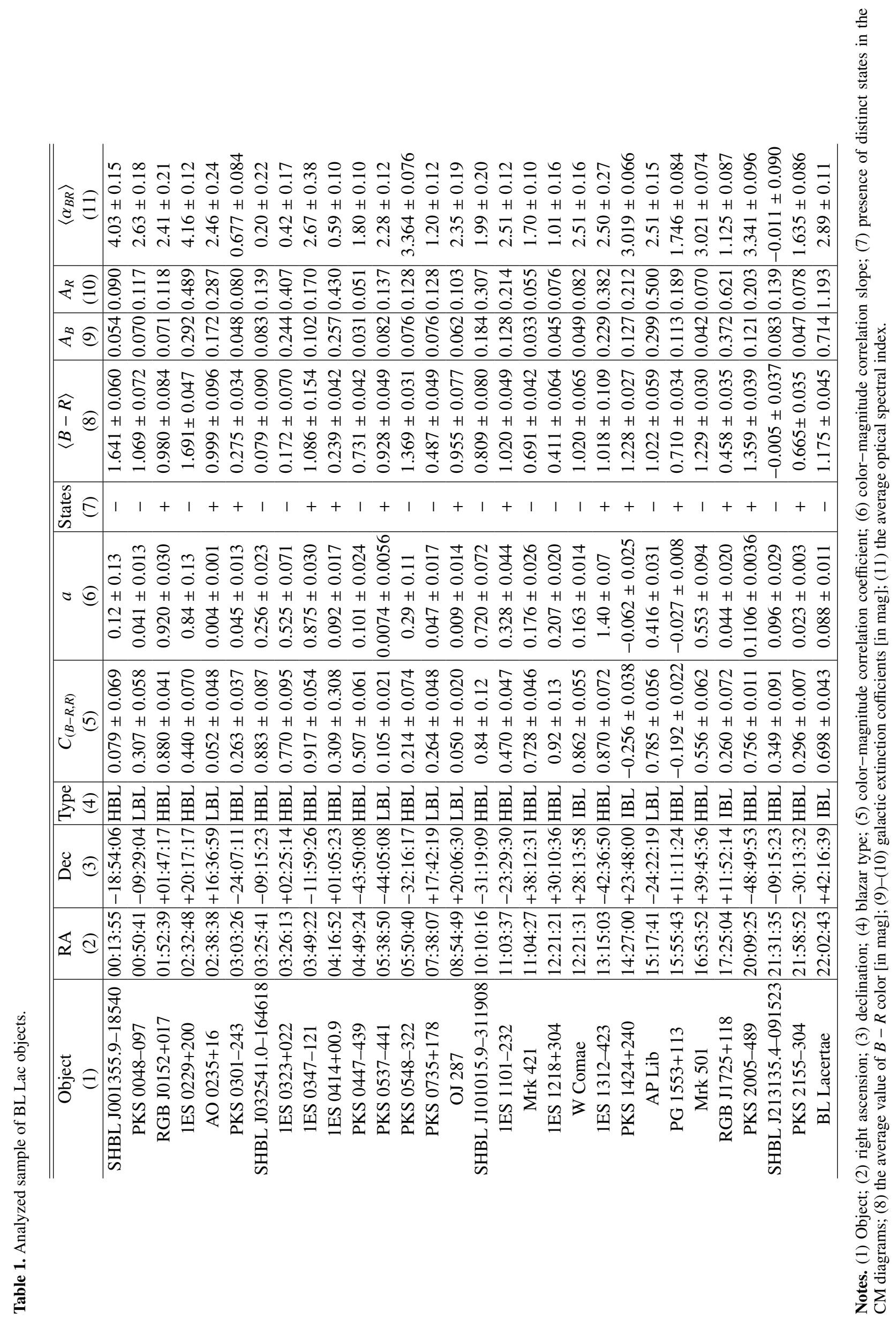


Table 2. ATOM observations.

\begin{tabular}{|c|c|c|c|c|c|c|}
\hline Object & 2007 & 2008 & 2009 & 2010 & 2011 & 2012 \\
\hline SHBL J001355.9-18540 & & 11,12 & $5-12$ & $8-12$ & $6-12$ & $5-12$ \\
\hline PKS 0048-097 & & & $8-12$ & $1,7-12$ & $6-12$ & $5-8,10$ \\
\hline RGB J0152+017 & 11,12 & $1,6-12$ & $1,6-12$ & $1,2,6-12$ & $1,6-12$ & $1,2,6-10$ \\
\hline 1ES $0229+200$ & $8-12$ & $1,8-12$ & $1,8-12$ & $8-11$ & $7-11$ & $7-10$ \\
\hline $\mathrm{AO} 0235+16$ & $1,9-11$ & $7,9-12$ & $1,7,8$ & 8 & & \\
\hline PKS 0301-243 & & & $8-12$ & $2,3,6-12$ & $7-12$ & $2,7-12$ \\
\hline SHBL J032541.0-164618 & & $9-12$ & $1,7-10,12$ & $3,7,8$ & $7,9,10,12$ & 1,7 \\
\hline 1ES $0323+022$ & & $10-12$ & $1,7-10,12$ & $2,9-12$ & $9-11$ & $1,7-10$ \\
\hline 1ES $0347-121$ & $8-12$ & $1,8-12$ & $1,3,7-12$ & $3,7,8-11$ & $1,7-11$ & $1,2,10$ \\
\hline 1ES $0414+00.9$ & $1,2,8-12$ & $1,8-12$ & $1,3,7-12$ & $2,8-12$ & $1,3,7-11$ & 10 \\
\hline PKS 0447-439 & & & 8,12 & $1-4,9-12$ & $1,4,8-11$ & $1-4,8,10$ \\
\hline PKS 0537-441 & & $10-12$ & $1,3-9$ & $1-5,9-12$ & $1,9-11$ & $1-4,8-10$ \\
\hline PKS 0548-322 & $5,9-12$ & $1,10-12$ & $1,3-5,7-12$ & $2-5,9-12$ & $1,2,4,5,9-11$ & $1-5,9,10$ \\
\hline PKS $0735+178$ & & $1,9-12$ & $1,2-5,9-12$ & $1,2-5,10-12$ & $1,2,4,5,9-11$ & $1,3-5$ \\
\hline OJ 287 & $2,5,11,12$ & $1,5,6,10-12$ & $1,2-6,10-12$ & $3-6,11,12$ & $1,3-6,11$ & $2,4,5,11,12$ \\
\hline SHBL J101015.9-311908 & 1 & $5,6,11,12$ & $2-7$ & $4-7$ & $1,3,4,6,7$ & \\
\hline 1ES 1101-232 & $2,6,7,11,12$ & $1,5-7,11,12$ & $1,3-7,11$ & $1,2-7,12$ & $1,4-6$ & $3-5,7$ \\
\hline Mrk 421 & 5 & $1,6,7,12$ & $1,3-6$ & 2 & & \\
\hline 1ES $1218+304$ & & & $4-6$ & $2,3-7$ & $3-6$ & $2-8$ \\
\hline W Comae & & 6,7 & $1,3-6$ & $2,4-7$ & $4-6$ & $3-5$ \\
\hline 1ES 1312-423 & & 9,12 & $1,3-8$ & $2-7$ & $1,2,4-8$ & \\
\hline PKS $1424+240$ & & & $6-8$ & $2-8$ & $4-8$ & $3-5$ \\
\hline AP Lib & & & & $6-8$ & $3-9$ & $2-9$ \\
\hline PG $1553+113$ & 8,9 & 8,9 & $2-9$ & $1,2-9$ & $4-9$ & $3,4-9$ \\
\hline Mrk 501 & $5,7,8$ & $5-7$ & $3-7,8$ & $6,7,9$ & 4,5 & 3,9 \\
\hline RGB J1725+118 & & 10 & $3-9$ & $6-9$ & $5-9$ & $3-9$ \\
\hline PKS 2005-489 & $5-12$ & $6-12$ & $3-12$ & $3-12$ & $5-11$ & $3-10$ \\
\hline SHBL J213135.4-091523 & & $7,9,10$ & $3-10$ & $4-8,10,11$ & $6-10$ & $4-7$ \\
\hline PKS 2155-304 & $5-12$ & $1,6-12$ & $1,3-12$ & $4-12$ & $5-11$ & $4-10$ \\
\hline BL Lacertae & & $8-11$ & $5-8,10$ & & & 6,9 \\
\hline
\end{tabular}

Notes. The table provides information about time periods (months and years) of the ATOM observations.

Figures 1 to 5 show the times of given measurements with the rainbow color scale normalized to the entire span of the ATOM observations of a given blazar. This color-coding demonstrates that the evaluated global correlation coefficients are only rarely reliable proxies of a persistent spectral evolution (in particular in the case of sources displaying a "clustering" of datapoints at different regions of the CM diagrams at distinct epochs; see Sect. 2.3 below). In addition, we note that the time and color scales for different objects in the analyzed sample are different, owing to the different total time spans and varying spacings of the analyzed ATOM observations (see Table 2). The result of the CM regression fits for the analyzed sample should therefore be taken with caution. All in all, however, significant positive $\mathrm{CM}$ correlations $\left(C_{(B-R, R)} \gg 0.5\right)$, indicating that the sources appear in general bluer when brighter, are found for 12 targets, while the negative (and, in fact, statistically insignificant) CM correlations are found for only two sources (PG 1553+113 and PKS 1424+240). Below we comment on each analyzed target individually.

\subsection{Notes on individual sources}

SHBL J001355.9-18540: the source was observed during 138 nights in 2008-2012. The collected data reveal no obvious CM correlations, but the source brightness varied only slightly, within the $\simeq 0.2 \mathrm{mag}$ range. The source optical spectrum was very steep, corresponding to $\alpha_{B R}>3.7$.

PKS 0048-097: the source was observed during 96 nights in 2009-2012. No clear CM relation found (in agreement with Ikejiri et al. 2011), even though the source brightness varied in a wide, $\sim 3$ mag range, and the optical spectral index oscillated between $\alpha_{B R} \sim 2$ and 3 .

RGB J0152+017: the source was observed during 269 nights in 2007-2012. Clear bluer-when-brighter behavior is present in the entire dataset, as well as in shorter isolated periods with the exception of the earliest measurements at the lowest flux level, when a weak redder-when-brighter trend could be noted. The observed flux changes were rather minor $(\simeq 0.4 \mathrm{mag})$, and the optical spectrum varied between $\alpha_{B R} \sim 2$ and 3 . 

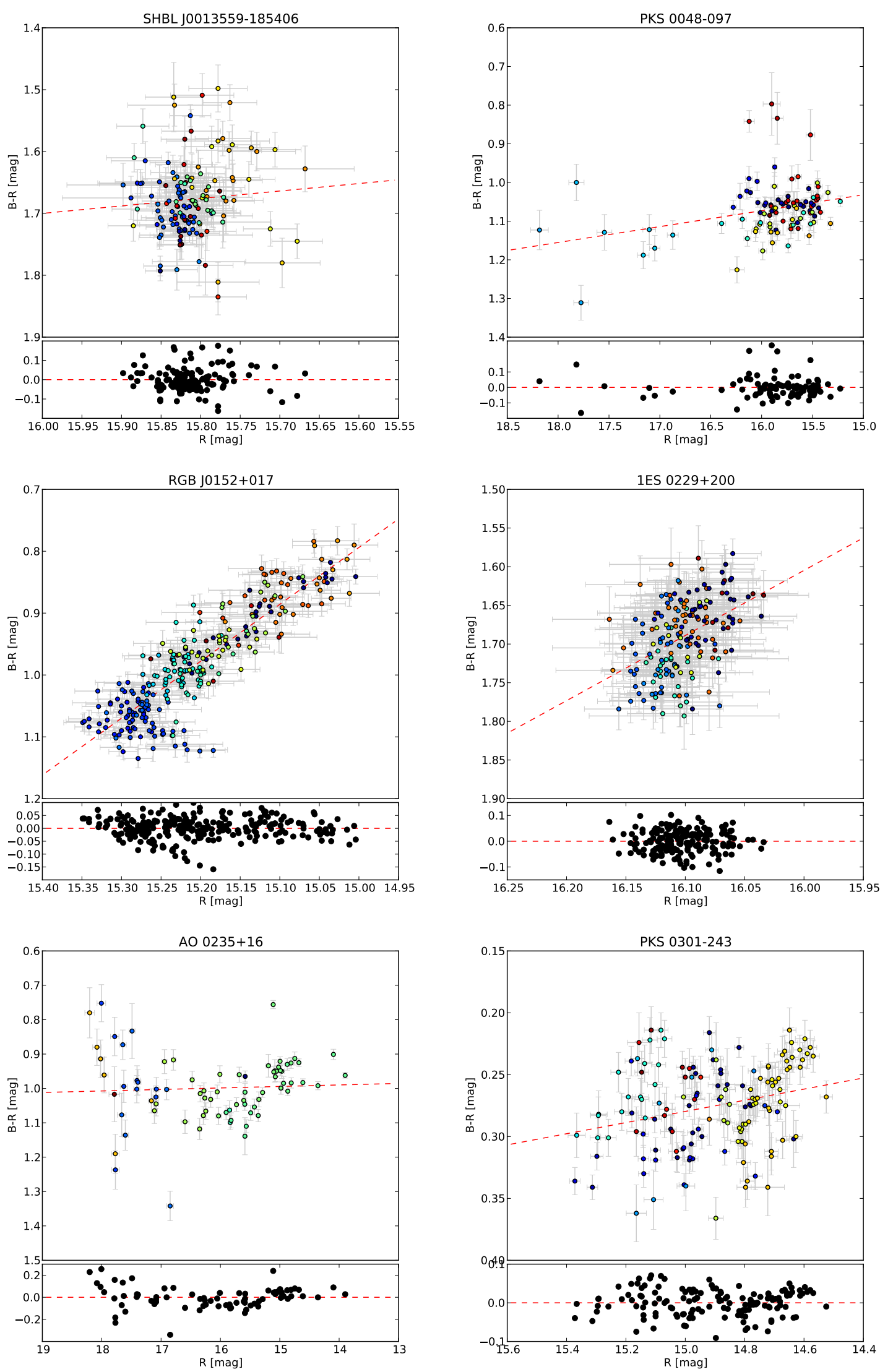

Fig. 1. $B-R$ color vs. $R$-band magnitude diagrams for SHBL J001355.9-18540, PKS 0048-097, RGB J0152+017, 1ES 0229+200, AO 0235+16, and PKS 0301-243. At each panel red dashed lines denote the fitted CM linear correlations (see Table 1 1 . Color of a given data point indicates the time of a given measurement. The earliest pointings are denoted by dark blue symbols and the most recent ones depicted in red, with the rainbow color scale normalized to the entire span of the ATOM observations of a given blazar. 

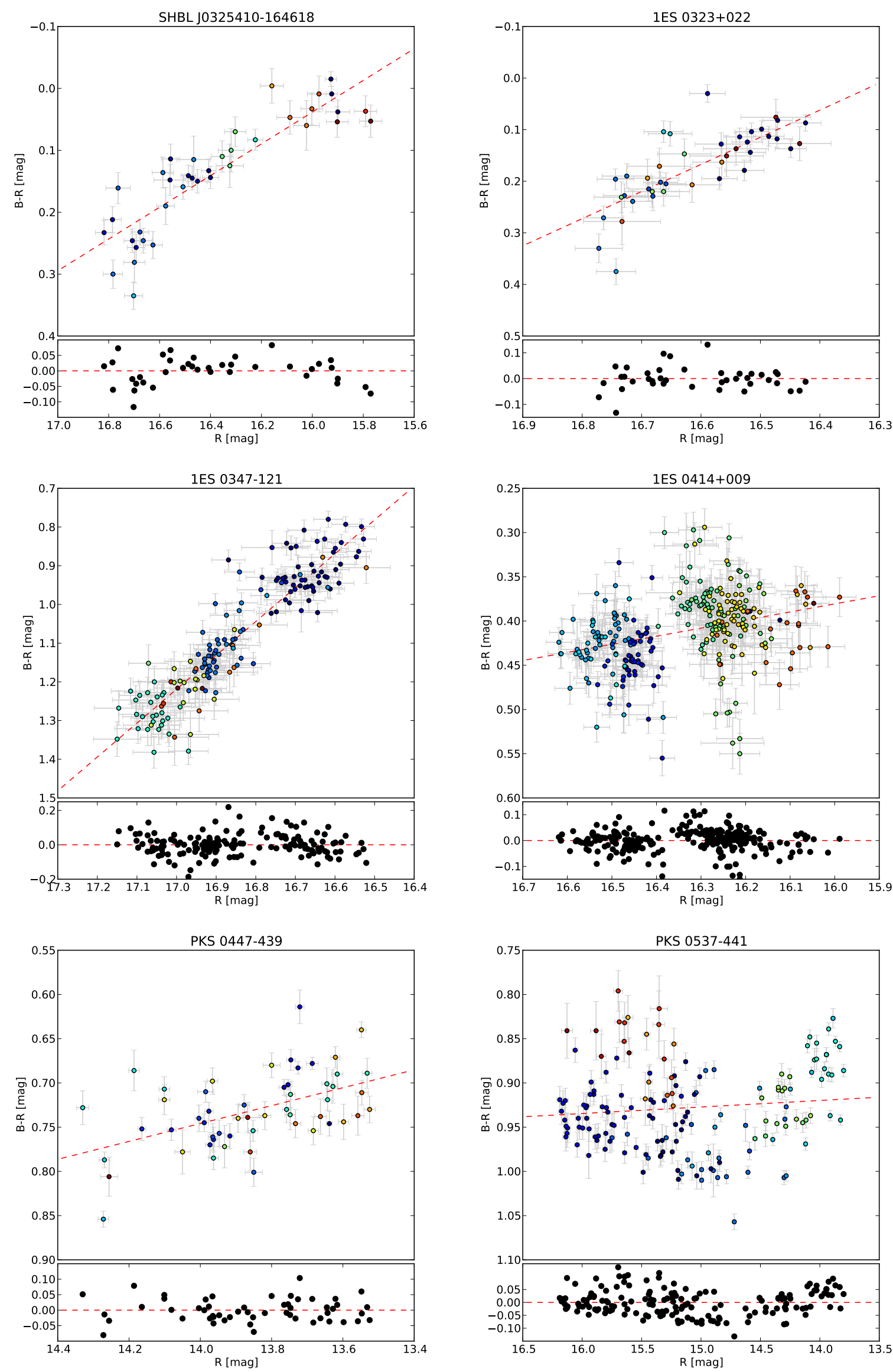

Fig. 2. Same as Fig. 1 for SHBL J032541.0-164618, 1ES 0323+022, 1ES 0347-121, 1ES 0414+00.9, PKS 0447-439, and PKS 0537-441. 
A. Wierzcholska et al.: Optical monitoring of BL Lac objects with ATOM
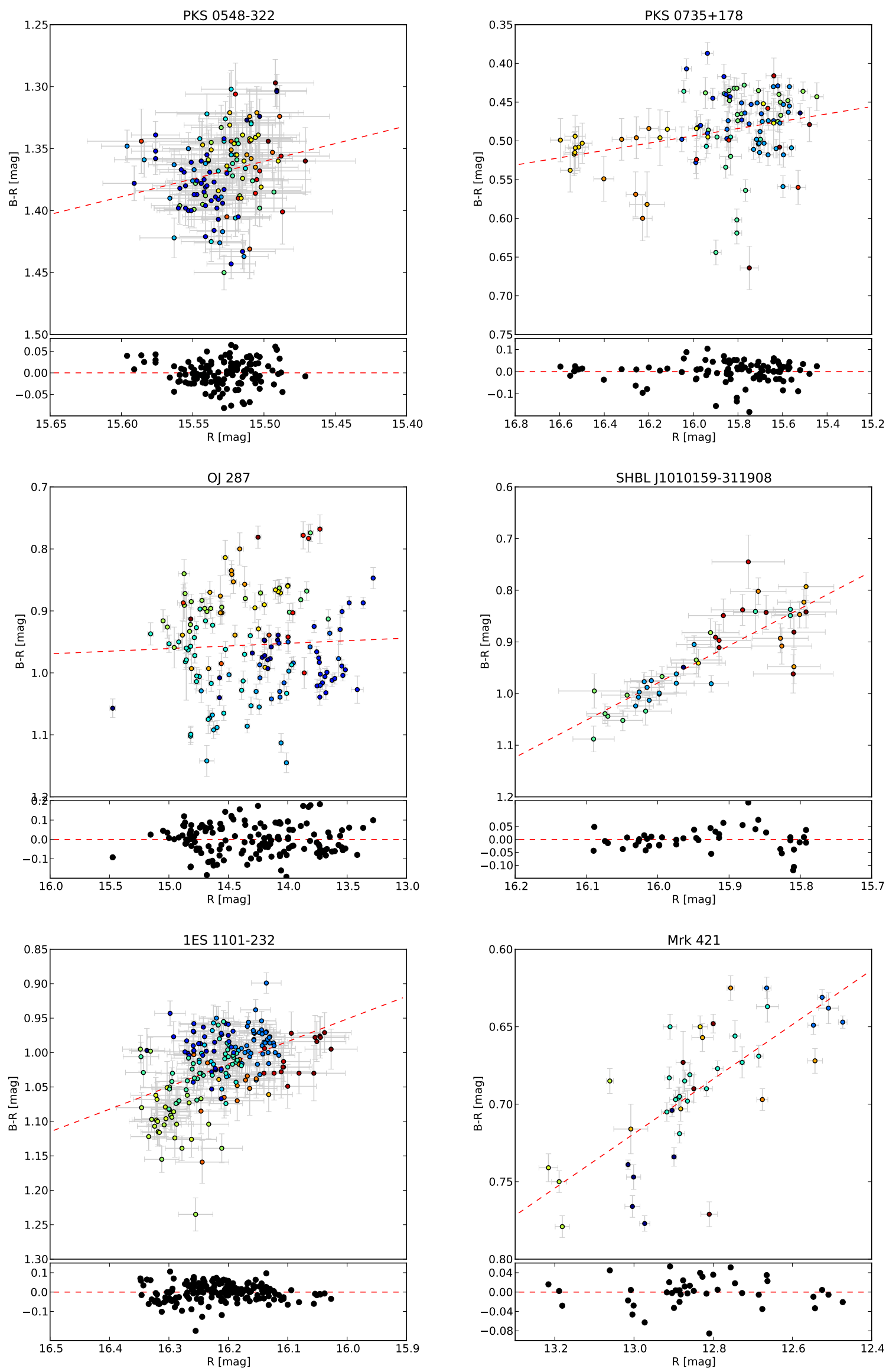

Fig. 3. Same as Fig. 1 for PKS 0548-322, PKS 0735+178, OJ 287, SHBL J101015.9-311908, 1ES 1101-232, and Markarian 421. 

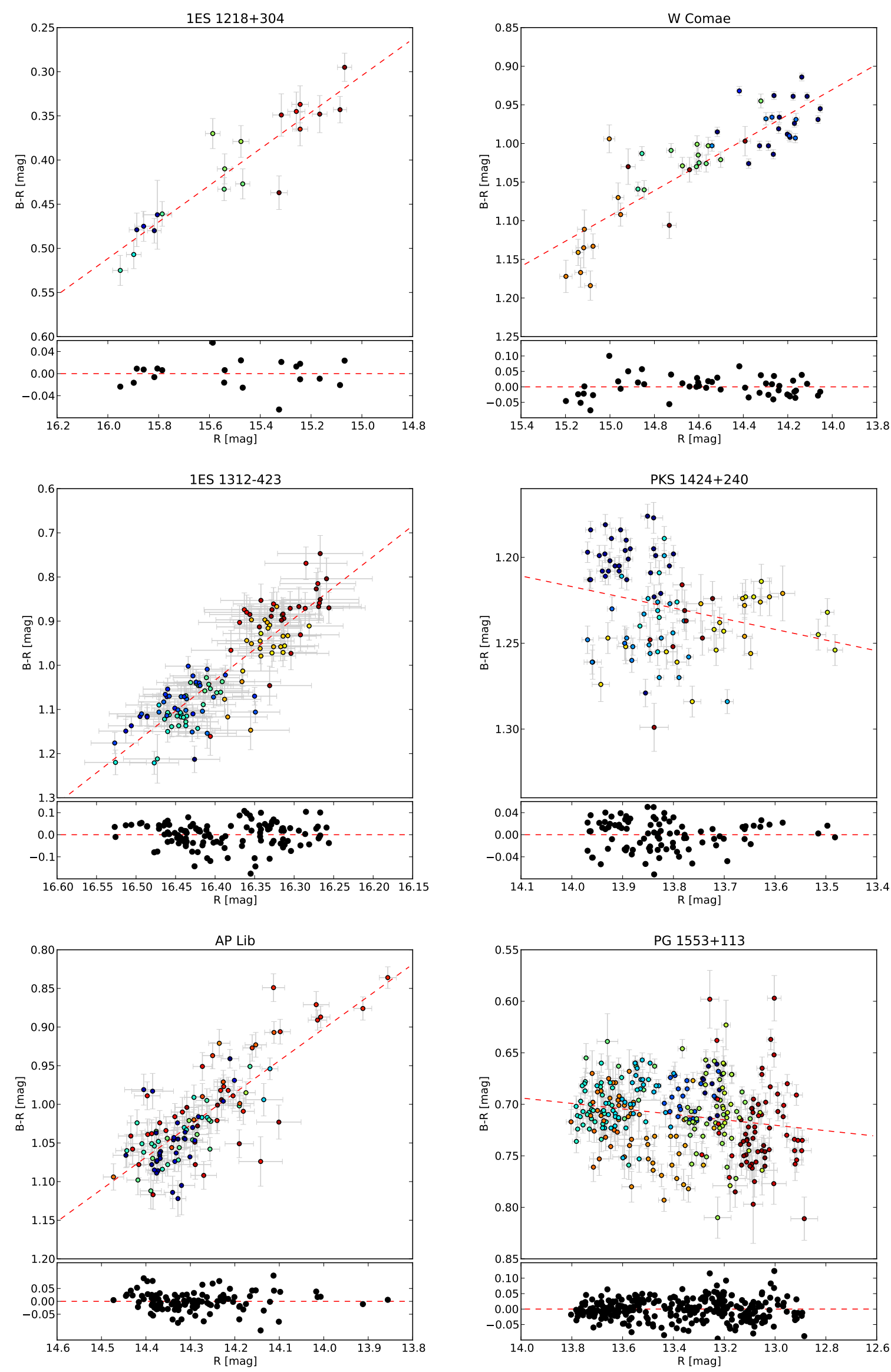

Fig. 4. Same as Fig. 1 for 1ES 1218+304, W Comae, 1ES 1312-423, PKS 1424+240, AP Librae, and PG 1553+113. 
A. Wierzcholska et al.: Optical monitoring of BL Lac objects with ATOM
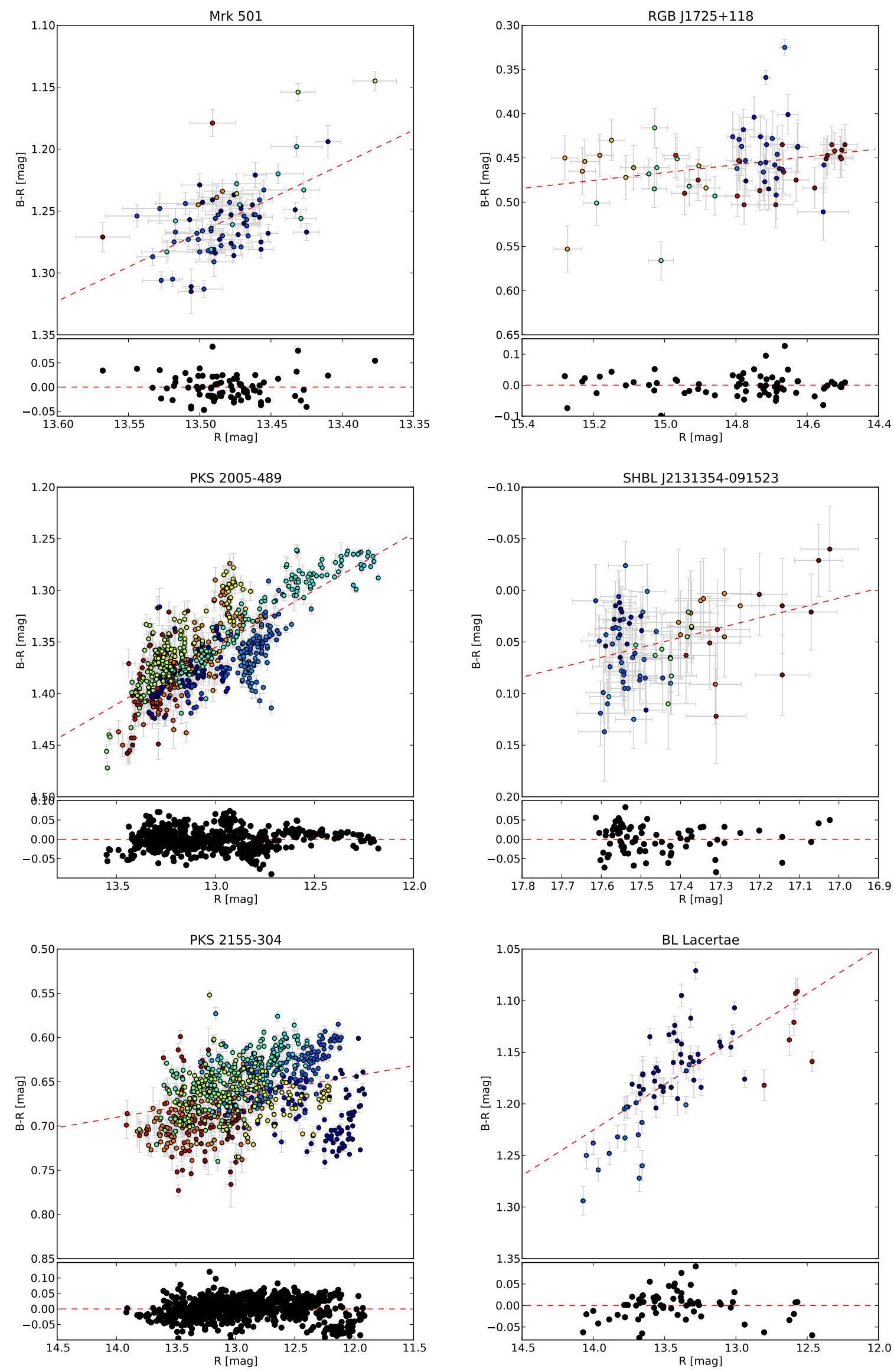

Fig. 5. Same as Fig. 1 for Markarian 501, RGB J1725+118, PKS 2005-489, SHBL J213135.4-091523, PKS 2155-304, and BL Lacertae. 
1ES 0229+200: the source was observed during 184 nights in 2007-2012. No clear CM correlation was found, either in the entire dataset or in shorter (e.g., one-year) intervals, but the source brightness varied only slightly within the $\simeq 0.2$ mag range. The optical continuum was very steep, with $\alpha_{B R}>3.7$.

AO 0235+16: the source was observed during 77 nights in 2007-2010. No significant CM correlation can be noted in the entire dataset despite the wide flux range covered ( $\sim 4 \mathrm{mag}$ ), although during the flaring phase at the highest flux level clear bluer-when-brighter chromatism is present. Ikejiri et al. (2011) and Raiteri et al. (2001) found significant CM correlations for this blazar, also during its quiescence. The observed spectral variations covered the range from $\alpha_{B R} \sim 2$ up to 3 .

PKS 0301-243: the source was observed during 145 nights in 2009-2012. The observed bluer-when-brighter trend in the entire dataset is rather weak, although during the selected shorter time intervals strong CM correlations can be noted, as shown in the additional Fig. 6. The flux varied by $\sim 1 \mathrm{mag}$, and the optical spectral index clustered within a relatively narrow range $0.5 \lesssim \alpha_{B R} \lesssim 1$.

SHBL J032541.0-164618: the source was observed during 38 nights in 2007-2011, revealing a flat optical spectrum $0 \lesssim$ $\alpha_{B R} \lesssim 0.7$ and a significant positive CM correlation within the $\sim 1$ mag flux range covered.

1ES 0323+022: the source was observed during 40 nights in 2008-2012, revealing clear bluer-when-brighter behavior despite only modest flux changes $(\simeq 0.4 \mathrm{mag})$, in agreement with the results of Ikejiri et al. (2011). The source spectrum varied between $\alpha_{B R} \sim 0$ and 1 .

1ES 0347-121: the source was observed during 161 nights in 2007-2012, revealing strong bluer-when-brighter chromatism within the $\sim 1$ mag flux range covered and optical spectral index changing from $\alpha_{B R} \sim 2$ up to 3.5.

1ES 0414+00.9: the source was observed during 285 nights in 2007-2012. The linear fit to all the collected datapoints does not show any clear CM correlation, but distinct states on the CM diagram can be noted. The flux varied within the $\lesssim 1$ mag range, and the spectral index oscillated between $\alpha_{B R} \sim 0.7$ and 1.3.

PKS 0447-439: the source was observed during 54 nights in 2009-2012, revealing only weak bluer-when-brighter chromatism in the $\sim 1$ mag flux variation range covered. The optical slope varied only slightly between $\alpha_{B R} \sim 1.5$ and 2 .

PKS 0537-441: the source was observed during 160 nights in 2008-2012. The analysis of long-term monitoring does not reveal any global CM correlation pattern; however, at low flux levels some hints for redder-when-brighter behavior can be noted, while at high flux levels a bluer-when-brighter trend seems to be present. Despite large-amplitude flux variations $(\sim 3 \mathrm{mag})$, the optical slope varied only slightly between $\alpha_{B R} \sim 2$ and 2.5 .

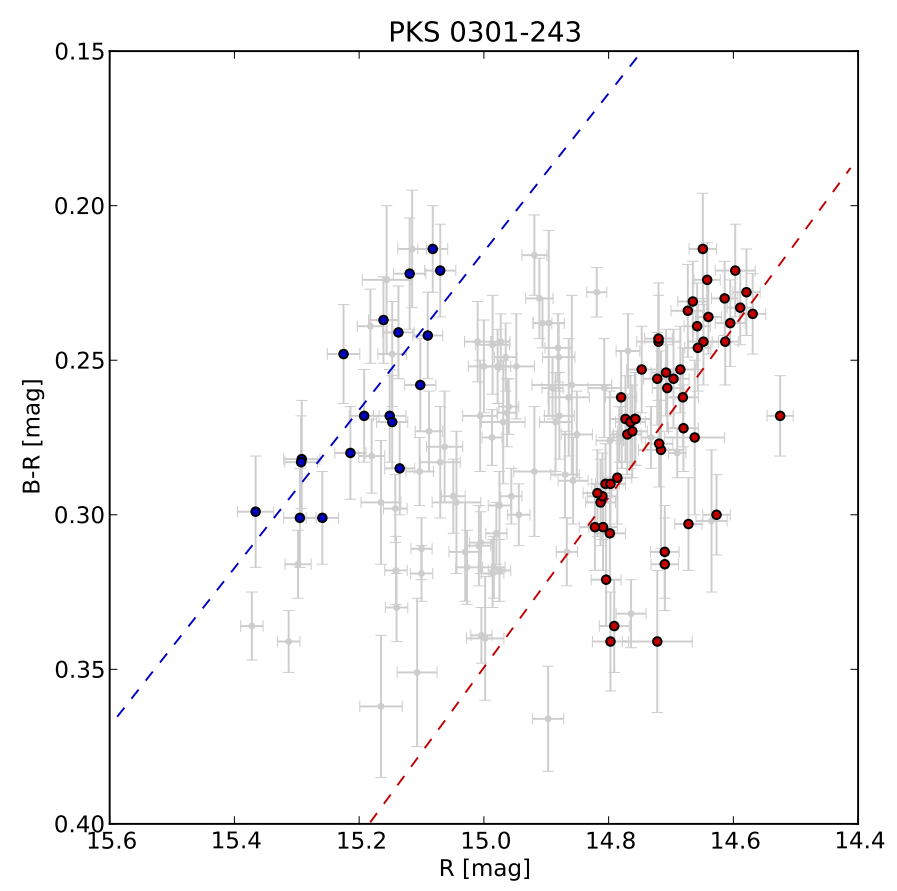

Fig. 6. $B-R$ vs. $R$ diagram for PKS 0301-243 with the two selected epochs corresponding to the two distinct optical states of the source (red and blue symbols, respectively).

PKS 0548-322: the source was observed during 132 nights in 2007-2012, revealing almost constant flux with $\simeq 0.1$ mag variations and persistently steep spectrum $\alpha_{B R} \simeq 3.5$.

PKS 0735+178: the source was observed during 104 nights in 2008-2012. No obvious global CM correlations were found; previously, some hints of bluer-when-brighter behavior of the blazar were reported by Gu et al. (2006). The observed flux variations covered the $\gtrsim 1$ mag range, and the optical slope varied between $\alpha_{B R} \sim 1$ and 1.5.

OJ 287: the source was observed during 148 nights in 20072012. No global CM correlations can be noted in the longterm light curve, although bluer-when-brighter chromatism can be seen in shorter isolated intervals, in agreement with the previous results presented in the literature (e.g., Carini et al. 1992, Dai et al. 2011; Ikejiri et al. 2011). The source displayed large, $\sim 2$ mag flux variations and spectral indices from $\alpha_{B R} \sim 2$ to 3 .

SHBL J101015.9-311908: the source was observed during 45 nights in 2008-2012, revealing significant positive CM correlation in the relatively narrow flux range covered $(\simeq 0.3 \mathrm{mag})$, with spectral indices $\alpha_{B R} \sim 2-3$.

1ES 1101-232: the source was observed during 197 nights in 2007-2012, showing only modest flux variations ( $\sim 0.3 \mathrm{mag})$ and weak chromatism with optical slope changing between $\alpha_{B R} \sim 2$ and 3 .

Markarian 421: the source was observed during 42 nights in 2007-2012. The overall bluer-when-brighter behavior is present, in agreement with the results by Ikejiri et al. (2011). 
About $\sim 1$ mag flux variations corresponded to the $\alpha_{B R} \sim 1.5-2.0$ range covered.

1ES 1218+304: the source was observed during only 20 nights in 2009, 2010, and 2012. Even though the ATOM observations of the blazar are limited, a strong CM correlation is clearly detected, both in the entire dataset and in shorter time intervals. About $\sim 1$ mag flux variations corresponded to the $\alpha_{B R} \sim 0.7-1.3$ range covered.

W Comae: the source was observed during 50 nights in 2008-2012, revealing strong bluer-when-brighter chromatism for $\sim 1$ mag flux variations and the optical slope changing from $\alpha_{B R} \sim 2$ up to 3 .

1ES 1312-423: the source was observed during 122 nights in 2008-2011. A strong positive CM correlation can be noted despite very limited $(\sim 0.25 \mathrm{mag})$ flux changes. The optical slope varied between $\alpha_{B R} \sim 2$ and 3 .

PKS 1424+240: the source was observed during 94 nights in 2009-2012. While the general regression fit returns a negative $\mathrm{CM}$ correlation coefficient, the analysis of shorter intervals reveals some hints of bluer-when-brighter behavior. The observed flux variations were only models $(\simeq 0.5 \mathrm{mag})$, and the optical spectrum remained basically constant, $\alpha_{B R} \sim 3$.

AP Librae: the source was observed during 112 nights in 20102012. Significant positive CM correlation was detected in the entire dataset and also on shorter (one-year-long) intervals. Modest, $\lesssim 1$ mag flux variations corresponded to the $\alpha_{B R} \sim 2-3$ range covered.

PG 1553+113: the source was observed during 294 nights in 2007-2012. The regression fit indicates negative though statistically insignificant CM correlation, in agreement with the results by Ikejiri et al. (2011); however, on shorter time intervals distinct states following redder-when-brighter or bluer-whenbrighter trends can be seen. About $\sim 1$ mag flux variations corresponded to the relatively narrow, $\alpha_{B R} \sim 1.5-2.0$ range covered.

Markarian 501: the source was observed during 79 nights in 2007-2012. Bluer-when-brighter behavior can be seen despite only weakly varying flux $(\simeq 0.2 \mathrm{mag})$, in agreement with the results by Ikejiri et al. (2011). The optical spectrum remained basically constant with $\alpha_{B R} \sim 3$.

RGB J1725+118: the source was observed during 69 nights in 2008-2012, revealing no obvious chromatism in the $\sim 1 \mathrm{mag}$ flux range covered, owing to the persistent optical spectrum with $\alpha_{B R} \sim 1$.

PKS 2005-489: the source was observed during 697 nights in 2007-2012. Significant positive CM correlations are seen in the entire dataset, as well as in shorter time intervals, which in addition seem to correspond to distinct spectral states of the blazar (each following bluer-when-brighter trend, though with different regression slopes). About $\sim 1.5$ mag flux variations corresponded to the relatively narrow, $\alpha_{B R} \sim 3.0-3.5$ range covered.
SHBL J213135.4-091523: the source was observed during 81 nights in 2008-2012, revealing only modest color changes with no obvious relation to the flux changes. The source spectrum remained very flat, $0 \lesssim \alpha_{B R}<0.5$, within the $\sim 0.6$ mag flux variation range.

PKS 2155-304: the source was observed during 792 nights in 2007-2012. Only weak bluer-when-brighter trend can be noted in the entire dataset, although at the highest flux level, two separate states of the source emerge, each characterized by a positive CM correlation but different optical colors (see Abramowski et al., in prep., for a further discussion). Large, $\sim 2$ mag flux variations corresponded to the relatively narrow, $\alpha_{B R} \sim 1.3-1.8$ range covered.

BL Lacertae: the source was observed during 65 nights in 2008, 2009, and 2012. A positive CM correlation can be seen in the entire dataset, albeit with a large scatter (in agreement with Villata et al. 2002, 2004, Ikejiri et al. 2011). The observed flux changes were significant $(\sim 1.5 \mathrm{mag})$, and the optical spectrum varied from $\alpha_{B R} \sim 2$ up to 3 .

\subsection{Multiwavelengh analysis}

In this section we compare the optical properties of ATOM blazars with the high-energy (HE) $\gamma$-ray and high-frequency radio data on the selected targets. The $0.1-100 \mathrm{GeV} \gamma$-ray data are taken from the Second Catalog of Fermi-LAT Sources (2FGL; Nolan et al. 2012). The corresponding radio observations were carried out at $15 \mathrm{GHz}$ by the Owens Valley Radio Observatory (OVRO), which is the $40 \mathrm{~m}$ telescope dedicated to observe Fermi-LAT targets (Richards et al.2011). It should be noted that while the ATOM data presented in this paper are from 20072012, the 2FGL includes the LAT data collected during the first 24 months of the science phase of the Fermi mission that began on 2008 August 4. The analyzed radio data, on the other hand, were collected during the period 2008-2012 in the sources' visibility windows. The multiwavelength data discussed below can thus be considered as only "quasi-simultaneous".

To account for different activity states of the studied blazars in the error bars evaluated for fluxes, luminosities, and optical colors (Figs. 74 9), we consider both the variability range and the standard error of a given quantity, and then choose the higher value. In the case of ATOM data, the variability range is estimated as the minimum and maximum values of the flux in the light curve. For Fermi-LAT data minimum and maximum flux based on the Flux_History values are taken from 2FGL, excluding flux upper limits. If the Flux_History includes only the flux upper limits, the variability range is estimated by the standard error of the mean 2FGL flux. The optical and $\gamma$-ray luminosity errors are mainly driven by the distance error, therefore to not underestimate this uncertainty, the higher value of the standard luminosity error and the variability range is used.

First we investigate the mean luminosities of the blazars in the radio $(15 \mathrm{GHz})$, optical ( $R$ filter), and $\gamma$-ray (LAT) bands, which are all provided in Table 3 . The corresponding luminosity-luminosity relations are presented in the upper panel of Fig. 7. In the plot, different symbols are used to denote three different types of BL Lac objects included in the sample, HBLs, LBLs, and LBLs. As shown in the figure, the luminosities obey almost linear correlations, which may either be intrinsic or induced by the redshift dependance (see in this context Ghirlanda 

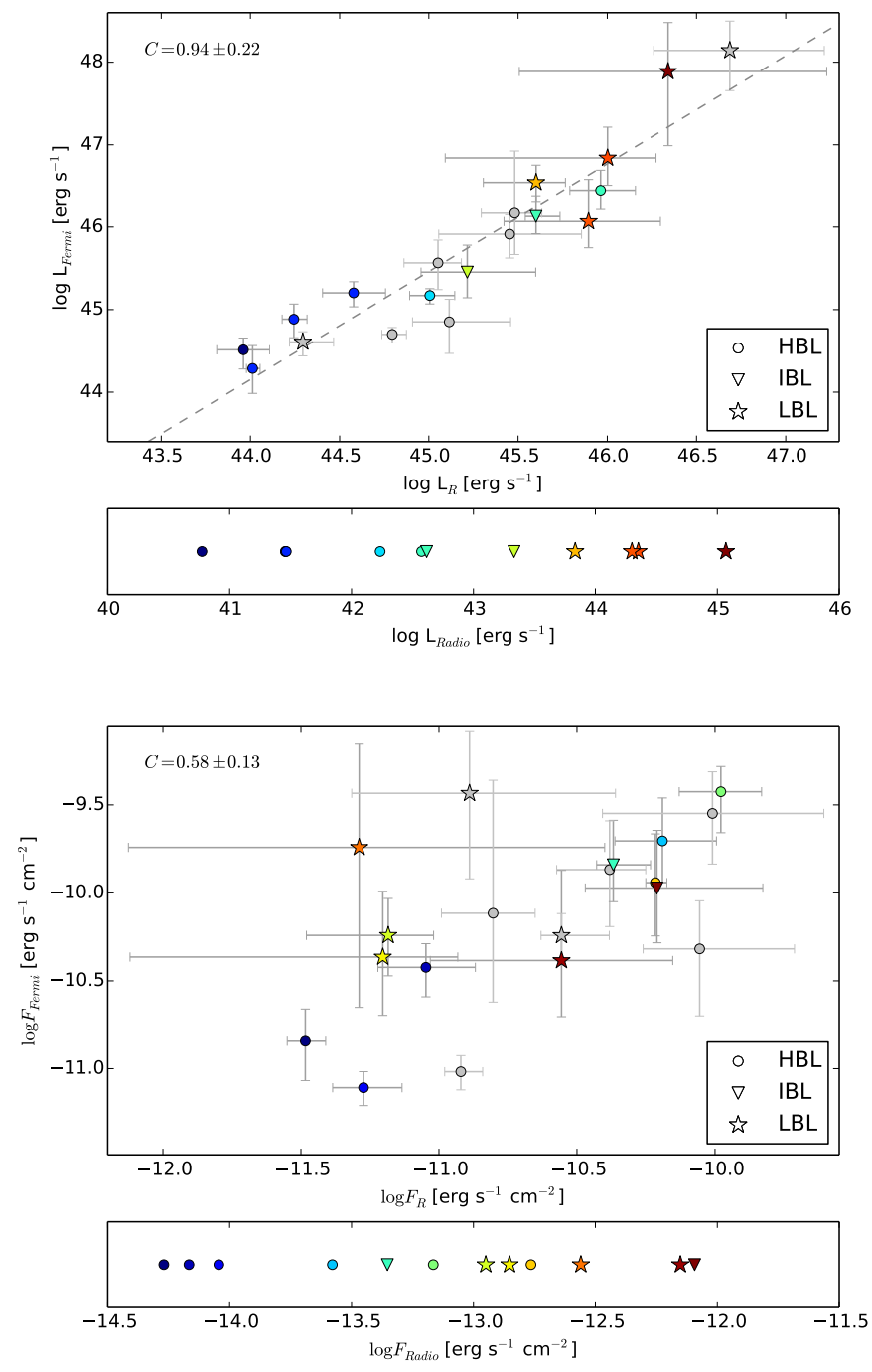

Fig. 7. Comparison between the mean HE $\gamma$-ray (LAT) and optical ( $R$ filter) luminosities (upper panel) and fluxes (lower panel) for the analyzed BL Lac objects (see Table 3 ). In each diagram, the color-coding denotes the luminosity or energy flux in the radio band $(15 \mathrm{GHz})$, depicted in the stripes below the panels. If there is no corresponding radio data, gray is used. Different symbols here are used to denote three different types of BL Lac objects included in the sample, namely HBLs (circles), LBLs (triangles), and LBLs (stars). The formally evaluated correlation coefficients $C$ are provided in the upper left corners of each panel. The error bars are evaluated as described in Sect. 2.4

et al. 2011; Arshakian et al. 2012; Hovatta et al. 2014). Since our blazar sample is scarce and incomplete, and also since we are not considering upper limits for those objects not included in the 2FGL, we cannot apply any well-posted statistical analysis to address this issue in detail. Instead, we simply investigate the corresponding flux-flux correlations, which all appear weaker than the luminosity-luminosity correlations (see the lower panel of Fig. 7). We therefore conclude that the observed linear luminosity-luminosity correlations are affected, at least to some extent, by the redshift dependence. In addition, nonsimultaneousness of the analyzed multifrequency dataset should be kept in mind in this context, since the comparison between mean fluxes in different bands depends on the activity state of a source during the analyzed period of time.
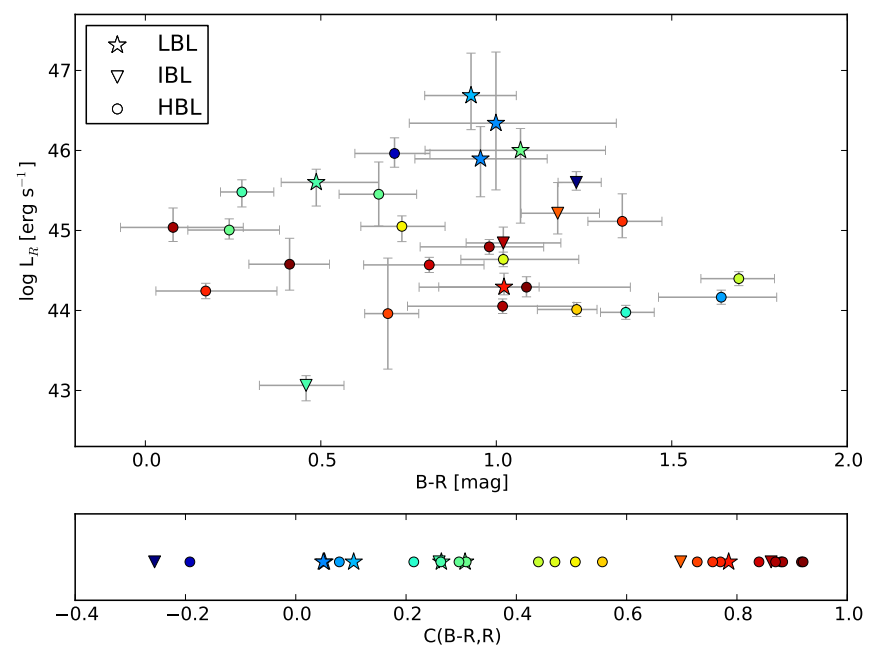

Fig. 8. Comparison between mean optical ( $R$ filter) luminosities of the analyzed BL Lac objects and their averaged optical colors $\langle B-R\rangle$ (see Table 3). In the diagram, the color-coding denotes the Pearson's correlation coefficient of the optical CM relation, $C_{(B-R, R)}$, as depicted in the stripe below the panel. Different symbols here are used to denote three different types of BL Lac objects included in the sample, namely HBLs (circles), LBLs (triangles), and LBLs (stars). The error bars are evaluated as described in Sect.2.4

Despite all these caveats, we note, however, that, as expected, LBLs are in general more luminous than HBLs, with IBL objects falling in between these two types of blazars. Nonetheless. interesting outliers can be spotted. In particular, the IBLclassified RGB J1725+118 is characterized by extremely low radio and optical luminosities, and this object does not posses any $\gamma$-ray counterpart in the 2FGL. On the other hand, the HBLclassified PG $1553+113$ is characterized by surprisingly high optical and $\gamma$-ray luminosities, as shown in the $\gamma$-ray/optical luminosity plot (upper panel in Fig. 7). In the same plot one can also see that AP Librae, an LBL, seems particularly underluminous for its type. In Sect. 3 we comment in more detail on the two interesting cases of PG $1553+113$ and AP Librae.

The comparison of the source optical luminosities and the average $\langle B-R\rangle$ colors is presented in Fig. 8. The plot does not reveal any obvious correlation. One can, however, notice that in the case of HBL type blazars, the $\langle B-R\rangle$ parameter covers a wide range from 0 mag to $1.7 \mathrm{mag}$, which translates to the huge range of average spectral indices from $\left\langle\alpha_{B R}\right\rangle \sim 0$ up to $\sim 4$. In the case of the two other subclasses of BL Lac objects included in the sample, the occupied $\langle B-R\rangle$ ranges are narrower, but this may be solely due to a low number IBLs and LBLs in the gathered sample.

In Fig. 9 we plot the HE $\gamma$-ray photon index for the LATdetected sources in the sample versus their optical luminosities. The color-coding here corresponds to the values of the Pearson's correlation coefficient of the optical CM relation. The diagram, which again may be affected quite substantially by nonsimultaneousness of the considered LAT and ATOM datasets, reveals only a weak tendency for the low-power sources (mostly HBLs) to be characterized by more significant CM correlations and flatter HE $\gamma$-ray spectra when compared with the high-power sources: IBLs and LBLs, which typically do not exhibit any obvious global $\mathrm{CM}$ correlations in the optical band, and tend to have steeper $\gamma$-ray spectra. 


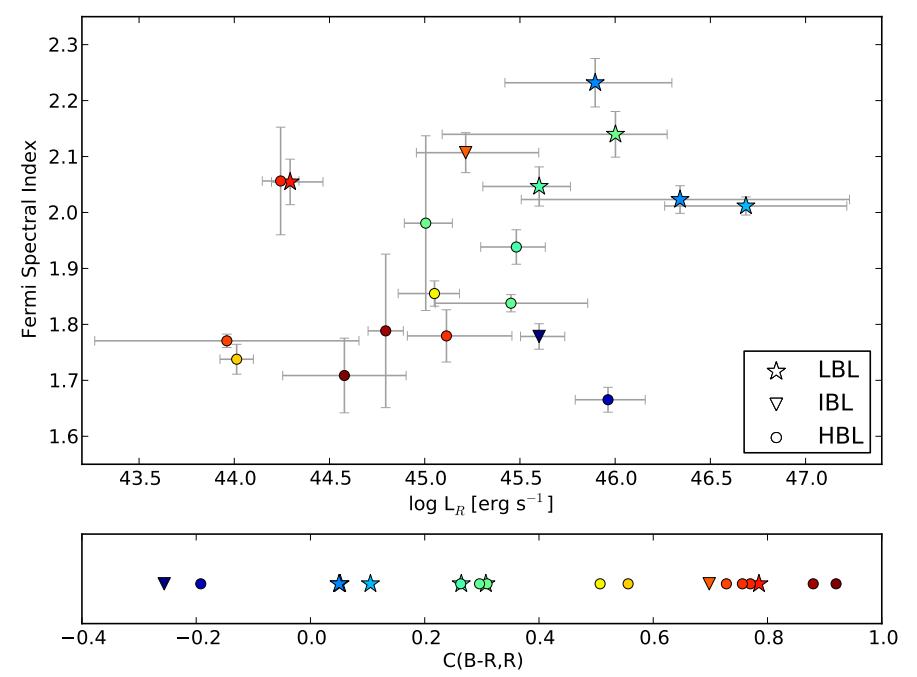

Fig. 9. Comparison between the HE $\gamma$-ray photon indices for the LATdetected sources in the sample and their optical luminosities. The color-coding denotes the Pearson's correlation coefficient of the optical CM relation, $C_{(B-R, R)}$, as depicted in the stripe below the diagram. Different symbols here are used to denote three types of BL Lac objects included in the sample: HBLs (circles), LBLs (triangles), and LBLs (stars). The error bars for $L_{R}$ are evaluated as described in Sect. 2.4 The error bars for the Fermi-LAT spectral indices are taken from 2FGL.

\section{Summary and discussion}

In this paper we analyzed the optical data for $30 \mathrm{BL}$ Lac type blazars collected using the ATOM telescope from 2007 until 2012. In particular, we studied the evolution of the selected sources on the color-magnitude diagrams in $B$ and $R$ filters. Clear "global" bluer-when-brighter trends are observed in the gathered datasets for 12 objects in the sample (with the Pearson's correlation coefficients $C_{(B-R, R)}>0.5$ ), including 1ES 0323+022, 1ES 0347+121, 1ES 1218+304, BL Lacertae, Mrk 421, PKS 2005-489, RGB J0152+117, SHBL J032541.0-164618, SHBL J101015.9-311908, W Comae, and 1ES 1312-423.

An important finding of the present analysis is the discovery of separate optical spectral states for several BL Lac objects. In the case of RGB J0152+017, AO 0235+16, PKS 0301-243, 1ES 0347-121, 1ES 0414+00.9, OJ 287, 1ES 1101-232, 1ES 1312-423, PKS 1424+240, PG 1553+113, RGB J1725+118, PKS 2005-489, and PKS 2155-304, the datapoints corresponding to different epochs of the source activity cluster in distinct, often isolated regions of the color-magnitude space. In some cases these distinct spectral states for a given source follow bluer-when-brighter trends, possibly (but not necessarily) with different correlation slopes or different optical colors, forming in this way separate "branches" on the CM diagrams (e.g., PKS 2155-304, PKS 0301-243). Presence of such distinct states results typically in the overall weakening of the global CM correlations, as observed for example in PKS 0301243. The analysis of all the available ATOM data for this blazar does not reveal any general bluer-when-brighter chromatism, even though during the selected shorter time intervals two distinct branches appear in the CM plot (see Fig. 6), separated by the $\simeq 0.4$ mag gap, but following similar bluer-whenbrighter tracks with the correlation coefficients $C_{(B-R, R)}=0.65$ and 0.79 , and with actually almost identical correlation slopes of $\simeq 0.26 \pm 0.05$.
The significance of bluer-when-brighter chromatism for the objects analyzed in this paper does not correlate with the optical color, but instead seems to depend (at least partly) on the blazar type, hence on the optical luminosity: low-power HBLs are typically characterized by significant positive CM correlations, while high-power LBLs typically display low values of the $C_{(B-R, R)}$ coefficients. Similar conclusions have been presented before by Ikejiri et al. (2011), even though these authors found positive CM correlations for almost all of the LBL type blazars included in their sample.

Ikejiri et al. (2011) claim in addition rather weak (or even absent) correlations between the optical flux and polarizationdegree changes in the studied sources and find that the lower luminosity objects generally show smaller amplitude variability (in flux, color, and polarization degree) when compared to the higher luminosity blazars. They conclude that the observed bluer-when-brighter behavior of blazars arises because shortterm (days or weeks) flat-spectrum flares are superimposed on slowly varying (months,years) or even stationary steep-spectrum (redder) components. It is important to note in this context that Ikejiri et al. did not correct the measured optical fluxes for a contamination from the starlight of host galaxies (which would require the hosts to be resolved well on optical images; e.g., Nilsson et al. 2007), or from the continuum emission of accretion disks. We did not correct for it in this study either.

In principle, a non-negligible contribution from accretion disks in luminous blazars - which are believed to accrete at high rates, and as such to be characterized by a prominent disk emission peaking at UV frequencies (e.g., Sbarrato et al. 2012) - may lead in some cases to the apparent redder-when-brighter evolution at optical frequencies, because the flat-spectrum disk emission may become less and less pronounced for the increasing steeper spectrum synchrotron emission of a blazar jet. This effect, however, is expected to play a role only in the case of FSRQs, or eventually for the most luminous LBLs. The starlight contamination due to host galaxies, on the other hand, may be relevant in low-accretion-rate blazars such as HBLs and IBLs.

Host galaxies of BL Lac objects are evolved giant ellipticals, for which the starlight emission peaks at near-infrared $(\sim 1 \mu \mathrm{m})$ wavelengths. Spectral features related to the starlight components are often seen directly in the composite SEDs of HBLs, especially during their quiescence phases (e.g., Mrk 501; see Abdo et al. 2011). The presence of these features may lead to the apparent bluer-when-brighter evolution at optical frequencies, since the steep-spectrum starlight emission may become less and less pronounced for the increasing flatter spectrum synchrotron emission of a blazar jet. And this may indeed be the case for at least some of the sources included in our sample. However, we argue that this effect cannot account for all the bluer-whenbrighter behavior seen in the collected ATOM dataset. That is because, as mentioned above, in many cases we observe separate "branches" in the CM diagrams, separated in flux or color but following similar bluer-when-brighter tracks (see, e.g., Fig. 6).

To investigate this issue in more detail, we divided the studied sample into the three subsamples of (i) eleven objects displaying only modest flux variations, $<1 \mathrm{mag}$; (ii) twelve sources with $R$-band fluxes changing by $\sim 1$ mag (a factor of a few); and (iii) seven blazars showing large-amplitude variability; $>1$ mag. The latter subsample is dominated by LBLs (PKS 0048-097, AO 0235+16, PKS 0537-441, and OJ 287), all of which do not display any clear chromatism, and are characterized by similarly steep optical slopes with $2<\alpha_{B R}<3$. The only two "highly variable" sources showing significant positive CM correlations are the HBL-classified PKS 2005-489 and the IBL-classified 


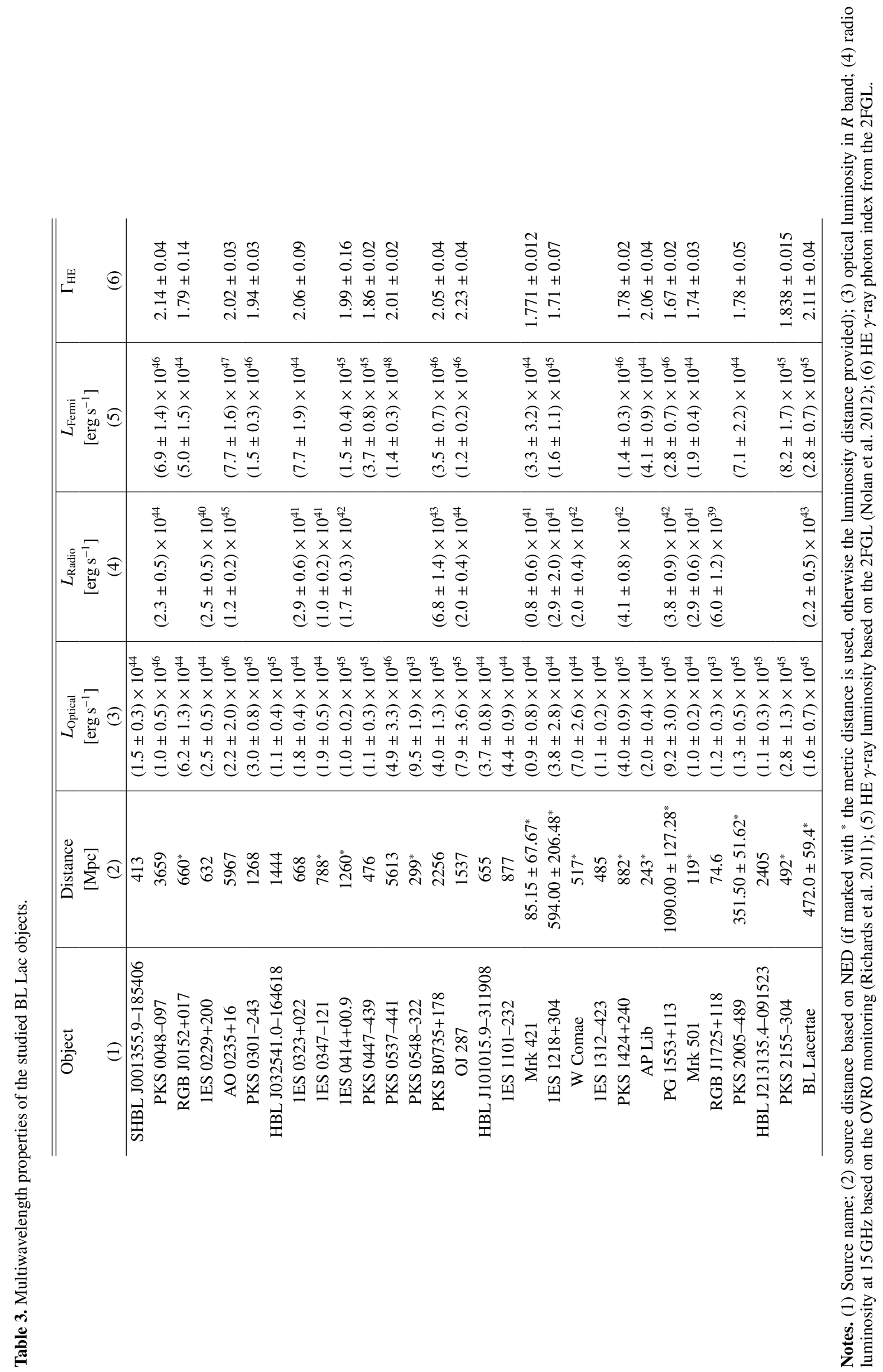


BL Lacertae. In their cases the observed bluer-when-brighter trends have to be intrinsic to the jets rather than resulting from the host-galaxy contribution. Interestingly, the remaining object in the subsample (iii), the HBL-classified PKS 2155-304, only displays weak chromatism but relatively flat spectrum $\alpha_{B R}<2$. The "moderately-variable" subsample (ii), on the other hand, is equally split into sources characterized by $C_{(B-R, R)}>0.5$ and $C_{(B-R, R)}<0.5$. The optical spectra of the blazars in this group are relatively flat, $0.5<\alpha_{B R}<2$, with the exception of steeper spectrum $\left(2<\alpha_{B R}<3\right)$ objects 1ES 0347-121 (HBL), W Comae (IBL), and AP Lib (LBL); these three "outliers" show particularly clear bluer-when-brighter trends. Finally, the majority of the objects from the "low-variable" sample (i) do not exhibit any significant positive CM correlations. Their ultrasteep $\left(\alpha_{B R}>3\right.$ in the case of SHBL J001355.9-18540, 1ES $0229+200$, and PKS 0548-322) or very steep, and in addition persistent $\left(\alpha_{B R} \sim 3\right.$ for PKS 1424+240 and Mrk 501), spectra signal non-negligible or even dominant host-galaxy contributions to the fluxes measured with ATOM. The prominent exception here is the flat-spectrum SHBL J213135.4-091523. Only four blazars in subsample (i) reveal positive CM correlations (RGB J0152+017, 1ES 0323+022, SHBL J101015.9-311908, and 1ES 1312-423), and these may be the examples where the observed bluer-when-brighter trends are due to the underlying host galaxy components as discussed in the previous paragraph.

In this paper we also compared the optical properties of ATOM blazars with the high-energy $\gamma$-ray and high-frequency radio data. We find that the radio, optical, and $\gamma$-ray luminosities of sources included in the sample obey almost linear correlations, which may be either intrinsic or induced by the redshift dependence. We did not find any correlation between the source luminosities and optical colors, or any HE spectral indices. All these multiwavelength comparisons may, however, be significantly affected by the lack of simultaneity in the analyzed dataset, as well as by a limited number of objects (especially of the IBL and LBL type) included in the studied sample.

In our analysis we noticed that two sources appear exceptional for their types: HBL-classified PG $1553+113$ is characterized by a particularly low value of the optical CM correlation coefficient and, at the same time, by surprisingly high optical and $\gamma$-ray luminosities despite the very flat $\mathrm{HE} \gamma$-ray continuum $\Gamma_{\mathrm{HE}} \simeq 1.67$ characteristic of an HBL; LBL-classified AP Librae, on the other hand, seems particularly underluminous for its type in optical and HE $\gamma$-rays and displays a significant positive CM correlation. Interestingly, the broad-band SED of AP Librae is indeed unusual, because it is characterized by a particularly broad high-energy hump in the SED representation (Kaufmann 2011). In contrast, one of the main characteristics of the broad-band SED of PG 1553+113 noticed in Aleksić et al. (2012) is the narrowness of its high-energy hump, which implies a relatively high value of the minimum electron energy within the dominant blazar emission zone.

\section{References}

Abdo, A. A., Ackermann, M., Agudo, I., et al. 2010, ApJ, 716, 30 Abdo, A. A., Ackermann, M., Ajello, M., et al. 2011, ApJ, 727, 129 Ackermann, M., Ajello, M., Allafort, A., et al. 2011, ApJ, 743, 171 Aharonian, F., Akhperjanian, A. G., Bazer-Bachi, A. R., et al. 2006, A\&A, 457, 899

Aharonian, F., Akhperjanian, A. G., Bazer-Bachi, A. R., et al. 2007, ApJ, 664, L71

Aleksić, J., Alvarez, E. A., Antonelli, L. A., et al. 2012, ApJ, 748, 46

Arshakian, T. G., León-Tavares, J., Böttcher, M., et al. 2012, A\&A, 537, A32

Atwood, W. B., Abdo, A. A., Ackermann, M., et al. 2009, ApJ, 697, 1071

Begelman, M. C., Blandford, R. D., \& Rees, M. J. 1984, Rev. Mod. Phys., 56, 255

Bessell, M. S. 1990, PASP, 102, 1181

Bessell, M. S., Castelli, F., \& Plez, B. 1998, A\&A, 333, 231

Bhatta, G., Webb, J. R., Hollingsworth, H., et al. 2013, A\&A, 558, A92

Bonning, E., Urry, C. M., Bailyn, C., et al. 2012, ApJ, 756, 13

Carini, M. T., Miller, H. R., Noble, J. C., \& Goodrich, B. D. 1992, AJ, 104, 15

Chatterjee, R., Bailyn, C. D., Bonning, E. W., et al. 2012, ApJ, 749, 191

Clements, S. D., \& Carini, M. T. 2001, AJ, 121, 90

Dai, Y., Wu, J., Zhu, Z.-H., Zhou, X., \& Ma, J. 2011, AJ, 141, 65

Dai, Y., Wu, J., Zhu, Z.-H., et al. 2013, ApJS, 204, 22

Dermer, C. D., \& Schlickeiser, R. 1993, ApJ, 416, 458

Fossati, G., Maraschi, L., Celotti, A., Comastri, A., \& Ghisellini, G. 1998, MNRAS, 299, 433

Gaur, H., Gupta, A. C., Wiita, P. J., et al. 2014, ApJ, 781, L4

Ghirlanda, G., Ghisellini, G., Tavecchio, F., Foschini, L., \& Bonnoli, G. 2011, MNRAS, 413, 852

Ghisellini, G., Villata, M., Raiteri, C. M., et al. 1997, A\&A, 327, 61

Ghosh, K. K., Ramsey, B. D., Sadun, A. C., \& Soundararajaperumal, S. 2000, ApJS, 127, 11

Gu, M. F., Lee, C.-U., Pak, S., Yim, H. S., \& Fletcher, A. B. 2006, A\&A, 450, 39

Hauser, M., Möllenhoff, C., Pühlhofer, G., et al. 2004, Astron. Nachr., 325, 659 Hovatta, T., Pavlidou, V., King, O. G., et al. 2014, MNRAS, 439, 690

Ikejiri, Y., Uemura, M., Sasada, M., et al. 2011, PASJ, 63, 639

Kaufmann, S. 2011, Int. Cosmic Ray Conf., 8, 199

Konigl, A. 1981, ApJ, 243, 700

Marscher, A. P., \& Gear, W. K. 1985, ApJ, 298, 114

Nilsson, K., Pasanen, M., Takalo, L. O., et al. 2007, A\&A, 475, 199

Nolan, P. L., Abdo, A. A., Ackermann, M., et al. 2012, ApJS, 199, 31

Padovani, P., \& Giommi, P. 1995, ApJ, 444, 567

Raiteri, C. M., Villata, M., Aller, H. D., et al. 2001, A\&A, 377, 396

Raiteri, C. M., Villata, M., D’Ammando, F., et al. 2013, MNRAS, 436, 1530

Richards, J. L., Max-Moerbeck, W., Pavlidou, V., et al. 2011, ApJS, 194, 29

Saito, S., Stawarz, Ł., Tanaka, Y. T., et al. 2013, ApJ, 766, L11

Sasada, M., Uemura, M., Arai, A., et al. 2008, PASJ, 60, L37

Sbarrato, T., Ghisellini, G., Maraschi, L., \& Colpi, M. 2012, MNRAS, 421, 1764

Schlafly, E. F., \& Finkbeiner, D. P. 2011, ApJ, 737, 103

Schlegel, D. J., Finkbeiner, D. P., \& Davis, M. 1998, ApJ, 500, 525

Sikora, M., Begelman, M. C., \& Rees, M. J. 1994, ApJ, 421, 153

Urry, C. M., \& Padovani, P. 1995, PASP, 107, 803

Villata, M., Raiteri, C. M., Kurtanidze, O. M., et al. 2002, A\&A, 390, 407

Villata, M., Raiteri, C. M., Kurtanidze, O. M., et al. 2004, A\&A, 421, 103

Wagner, S. J., \& Witzel, A. 1995, ARA\&A, 33, 163 